\title{
BMJ Open Association between serum uric acid and obesity in Chinese adults: a 9-year longitudinal data analysis
}

\author{
Jie Zeng (10 , ${ }^{1,2}$ Wayne R Lawrence, ${ }^{3}$ Jun Yang, ${ }^{4}$ Junzhang Tian, ${ }^{1}$ Cheng Li, ${ }^{5}$ \\ Wanmin Lian, ${ }^{6}$ Jingjun He, ${ }^{7}$ Hongying Qu, ${ }^{1,7}$ Xiaojie Wang, ${ }^{1}$ Hongmei Liu, ${ }^{2,8}$ \\ Guanming Li, ${ }^{1}$ Guowei $\mathrm{Li}^{1,9}$
}

To cite: Zeng J, Lawrence WR Yang J, et al. Association between serum uric acid and obesity in Chinese adults: a 9-year longitudinal data analysis. BMJ Open 2021;11:e041919. doi:10.1136/ bmjopen-2020-041919

- Prepublication history and additional material for this paper is available online. To view these files, please visit the journal online (http://dx.doi.org/10. 1136/bmjopen-2020-041919).

Received 23 June 2020 Revised 14 November 2020 Accepted 25 November 2020

Check for updates

(c) Author(s) (or their employer(s)) 2021. Re-use permitted under CC BY-NC. No commercial re-use. See rights and permissions. Published by BMJ.

For numbered affiliations see end of article.

Correspondence to

Dr Guowei Li; liguowei099@126.com and

Dr Guanming Li;

lywergd@163.com

\section{ABSTRACT}

Objectives Hyperuricaemia has been reported to be significantly associated with risk of obesity. However, previous studies on the association between serum uric acid (SUA) and body mass index (BMI) yielded conflicting results. The present study examined the relationship between SUA and obesity among Chinese adults. Methods Data were collected at Guangdong Second Provincial General Hospital in Guangzhou City, China, between January 2010 and December 2018. Participants with $\geq 2$ medical check-up times were included in our analyses. Physical examinations and laboratory measurement variables were obtained from the medical check-up system. The high SUA level group was classified as participants with hyperuricaemia, and obesity was defined as $B M l \geq 28 \mathrm{~kg} / \mathrm{m}^{2}$. Logistic regression model was performed for data at baseline. For all participants, generalised estimation equation (GEE) model was used to assess the association between SUA and obesity, where the data were repeatedly measured over the 9-year study period. Subgroup analyses were performed by gender and age group. We calculated the cut-off values for SUA of obesity using the receiver operating characteristic curves (ROC) technique.

Results A total of 15959 participants (10 023 men and 5936 women) were included in this study, with an average age of 37.38 years (SD: 13.27) and average SUA of $367.05 \mu \mathrm{mol} / \mathrm{L}$ (SD: 97.97 ) at baseline, respectively. Finally, 1078 participants developed obesity over the 9year period. The prevalence of obesity was approximately $14.2 \%$ for high SUA level. In logistic regression analysis at baseline, we observed a positive association between SUA and risk of obesity: $\mathrm{OR}=1.84$ (95\% Cl: 1.77 to 1.90$)$ for per-SD increase in SUA. Considering repeated measures over 9 year for all participants in the GEE model, the perSD OR was 1.85 (95\% Cl: 1.77 to 1.91) for SUA and the increased risk of obesity were greater for men $(\mathrm{OR}=1.45)$ and elderly participants $(\mathrm{OR}=1.01)$. In subgroup analyses by gender and age, we observed significant associations between SUA and obesity with higher risk in women $(\mathrm{OR}=2.35)$ and young participants $(\mathrm{OR}=1.87)$ when compared with men $(\mathrm{OR}=1.70)$ and elderly participants $(\mathrm{OR}=1.48)$. The SUA cut-off points for risk of obesity using ROC curves were approximately consistent with the international standard.

Conclusions Our study observed higher SUA level was associated with increased risk of obesity. More

\section{Strengths and limitations of this study}

- This is the first large long-term medical check-up study to explore the relationship between serum uric acid and obesity in China.

- The study analysis was based on the generalised estimation equation model which can increase the accuracy of the prediction.

- The results from this study could inform prevention methods for obesity, especially in medically underserved areas where medical service is insufficient.

- The younger screening population in this study may underestimate the increased risk of uric acid among the elderly obese.

high-quality research is needed to further support these findings.

\section{INTRODUCTION}

An individual's health behaviour can influence both physical health and ability to recover from an illness. Annual medical check-up is an example of a positive health behaviour, as this preventative measure is associated with earlier disease detection, greater treatment success and faster recovery from a disease. ${ }^{1}$ For this reason, medical data obtained from primary care is a useful source as it includes information on symptoms and healthcare utilisation, all beneficial for use in prediction analysis. Medical check-up data often includes a variety of diagnostic tests to assess health status for early detection and disease prevention. Additionally, medical check-up data provides valuable information on present and past health conditions that are generally difficult to obtain in most population-based data. ${ }^{2}$ More specifically, medical check-up data is a reliable and objective measure for identifying chronic diseases such as hyperuricaemia and obesity.

Serum uric acid (SUA) is the final product of purine metabolism in humans, potentially 
resulting in hyperuricaemia. ${ }^{34}$ In China, the prevalence of hyperuricaemia is $13.3 \%$, with $19.4 \%$ for men and $7.9 \%$ for women. ${ }^{5}$ Additionally, in 2019 the obesity prevalence was nearing 12\% in China. Among obese patients, hyperuricaemia is commonly observed. Although changes in obesity was reported to be independently correlated with changes in uric acid concentration, there might be an interaction between them as suggested in prior pathophysiological and metabolic studies. ${ }^{6}$ Epidemiological and clinical evidence supports a strong significant positive association between SUA and obesity in the adult population of China, Japan, India, Pakistan and Iraq. ${ }^{7}$ A cross-sectional study showed that body mass index (BMI) significantly increases with elevated SUA among 27009 middle-aged and elderly Chinese adults. ${ }^{8}$ Previous research showed that hyperuricaemia can cause obesity by accelerating hepatic and peripheral lipogenesis. ${ }^{9}$ With the increasing prevalence of obesity among adults with hyperuricaemia, it is of public health importance to evaluate the long-term epidemiological transitions to develop policies centred on intervention.

Numerous trend analyses have reported the association between SUA and BMI based on short-term survey data in China. ${ }^{10}{ }^{11}$ However, there remains a gap in evidence regarding the long-term trend for providing estimates on the risks of obesity among Chinese adults during the last two decades. Therefore, the present study aimed to examine the relationship between SUA and risk of obesity using the 9-year medical check-up data among Chinese adults from 2010 to 2018.

\section{METHODS}

\section{Study design and subjects}

We conducted a large retrospective study in China. Medical examinations were performed in 2010 and 2018 at the Guangdong Second Provincial General Hospital in Guangzhou City, China (figure 1). Individuals were excluded from the study due to having (1) less than two medical checkups; (2) absence of blood biochemical examination; and (3) no documented information on BMI. Thus, a total of 15959 participants were included in the study analysis (figure 2).

\section{Measurements}

All participants were invited to join an in-person evaluation that included physical examination and laboratory testing. Physical examinations were conducted following a standardised protocol, including weight, height, waist circumference, hip circumference and blood pressure. Waist circumference was measured around the midway between the lowest border of the ribs and iliac crest in the horizontal plane. The quality of anthropometric data was confirmed by repeated measurements in the presence of researchers. Laboratory measurements were obtained to measure SUA, systolic blood pressure (SBP), diastolic blood pressure (DBP), total cholesterol (TC), triglycerides (TG), fasting plasma glucose (FPG), highdensity lipoprotein cholesterol (HDL-C), low-density lipoprotein cholesterol (LDL-C), creatinine $(\mathrm{Cr})$ and blood urea nitrogen (BUN).

\section{Outcomes and definitions}

Hyperuricaemia was defined as having SUA concentrations $>7.0 \mathrm{mg} / \mathrm{dL}(416.4 \mu \mathrm{mol} / \mathrm{L})$ in men or $>6.0 \mathrm{mg} / \mathrm{dL}$ $(356.9 \mathrm{\mu mol} / \mathrm{L})$ in women. ${ }^{12}{ }^{13}$ SUA levels were categorised into two groups (normal and high SUA) to compare the prevalence of obesity and its association with SUA. The high SUA level group was classified as participants with hyperuricaemia. BMI was defined as weight divided by height ${ }^{2}\left(\mathrm{~kg} / \mathrm{m}^{2}\right)$ and categorised into two groups (nonobese $\left(<28 \mathrm{~kg} / \mathrm{m}^{2}\right)$ and obese $\left.\left(\geq 28 \mathrm{~kg} / \mathrm{m}^{2}\right)\right)$ based on the
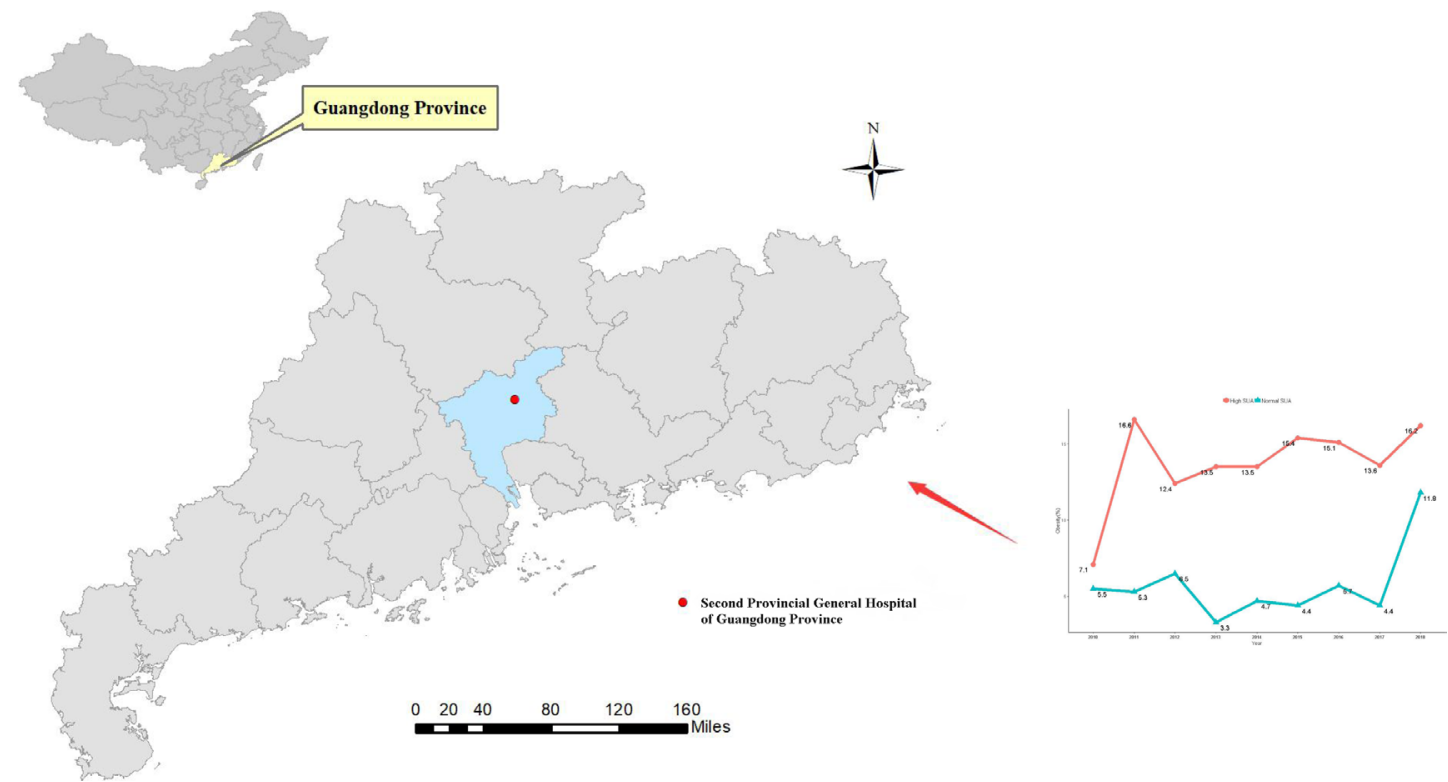

Figure 1 Location of Guangdong Second Provincial General Hospital (Guangzhou, Guangdong, China) and the prevalence of obesity by different years stratified by baseline serumuric acid. 


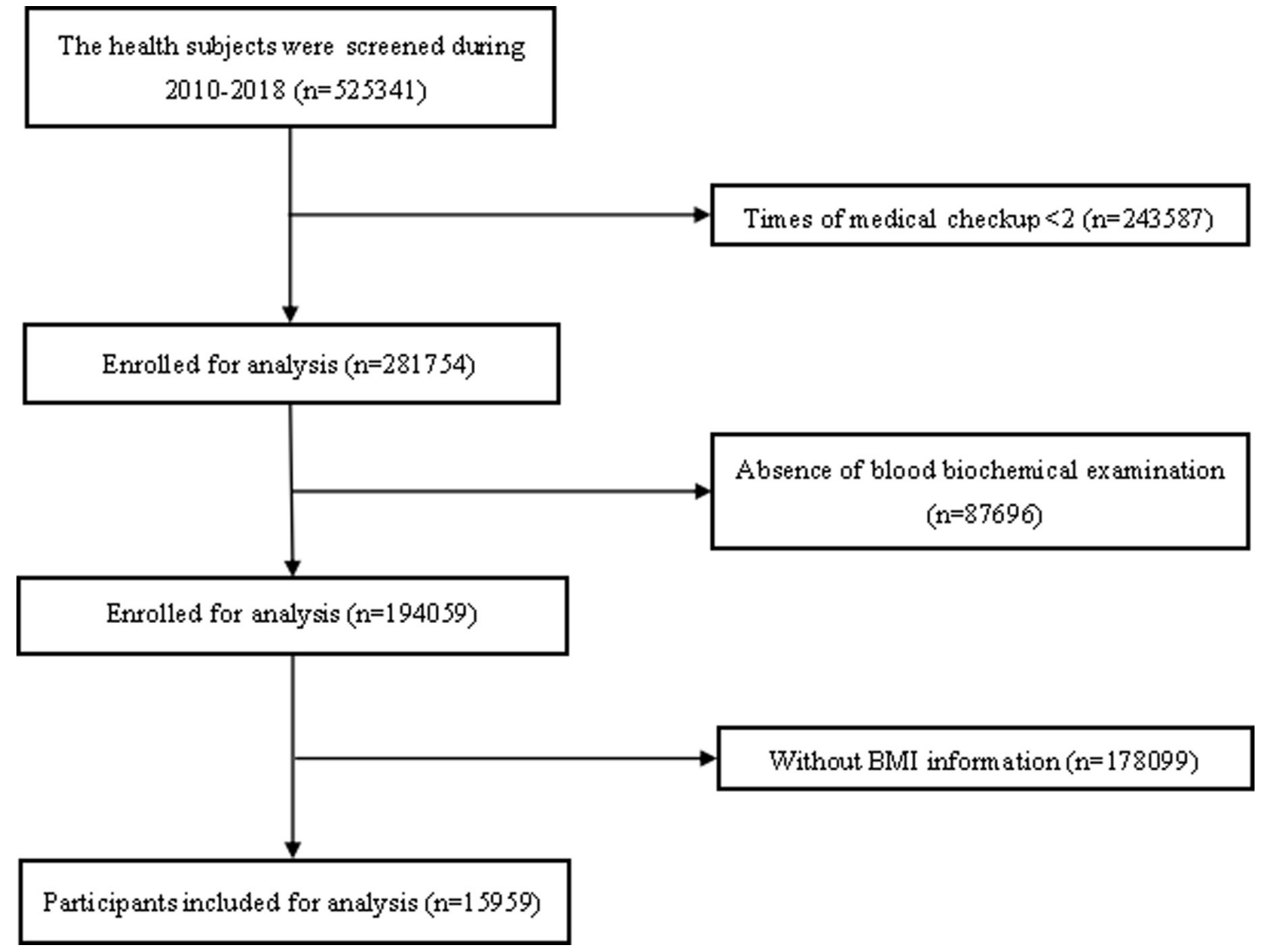

Figure 2 Flow diagram showing selection process of participants in our study. BMI, body mass index.

Asia-Pacific criteria set by the WHO. ${ }^{14}{ }^{15}$ We excluded patients taken drugs that might affect uric acid metabolism, such as losartan, furosemide and allopurinol.

\section{Statistical analysis}

We conducted descriptive analysis to present the characteristics of baselines participants. Continuous variables were reported as mean $\pm \mathrm{SD}$ and categorical variables as frequency and percentage, unless otherwise specified. Comparisons between two groups (obese and non-obese) were performed using Student's t-tests for continuous variables and $\chi^{2}$ analyses for categorical variables. Logistic regression model (LRM) was used to evaluate the relationship between risk of obesity and risk factors for the data at baseline. We also used generalised estimating equations (GEE) models with unstructured correlation structures to quantify their longitudinal association between SUA and risk of obesity ${ }^{16}$ given the data on SUA and obesity were repeatedly measured over the 9-year study period. All models were adjusted for age, gender, SBP, DBP, TC, TG, HDL-C, LDL-C, FPG, BUN and $\mathrm{Cr}$ in each group. Results were presented as OR and $95 \%$ CI with per $-1 \mu \mathrm{mol} / \mathrm{L}$ or per-SD increase in SUA.

We performed subgroup analyses using GEE models by: (1) gender (male vs female); and (2) age group (youth $<65$ years vs elderly $\geq 65$ years). Additionally, we calculated the cut-off values of SUA for risk of obesity using the receiver operating characteristic (ROC) curves, based on criteria including (1) the point on the curve with minimum distance from the left upper corner of the unit square; and (2) the point where the Youden's index is maximum. ${ }^{17}$ A two-sided $p$ value less than 0.05 was considered as the statistically significant. Analyses were performed using R V.3.5.3 (R Foundation for Statistical Computing, Vienna, Austria).

\section{Patient and public involvement}

There were no patient and/or public involvement in the design of this study.

\section{RESULTS}

There were 15959 participants (10 023 men) included in this study. The average number of health check-up for each participant was 2.62. Participants had a mean age of 37.38 years (SD: 13.27) and a mean SUA of 367.05 $\mu \mathrm{mol} / \mathrm{L}$ (SD: 97.97) at baseline, respectively. There were $1227(7.6 \%)$ participants that were obese at baseline. Significant differences between the obese and non-obese groups were observed for SUA, age, gender, SBP, DBP, TC, TG, HDL-C, LDL-C, FPG, BUN and Cr ( $p$ value $<0.001$ ) (table 1 ). In total, the prevalence of obesity was approximately $14.2 \%$ for high SUA level. Obesity prevalence significantly increased with elevating SUA in the subgroup analysis by gender and age group ( $p$ value $<0.001)$. The prevalence was higher in men than women. However, the prevalence had no obvious trend by age group (table 2 ). The prevalence of obesity significantly 
Table 1 Baseline characteristics and comparison between obesity and non-obesity participants

\begin{tabular}{|c|c|c|c|c|}
\hline \multirow[b]{2}{*}{ Characteristics } & All patients & Obesity* & Non-obesity & \multirow[b]{2}{*}{ P value } \\
\hline & $n=15959$ & $n=1227$ & $n=14732$ & \\
\hline SUA ( $\mu \mathrm{mol} / \mathrm{L})(\mathrm{SD})$ & 367.05 (97.97) & 434.95 (97.65) & 361.32 (95.82) & $<0.001$ \\
\hline Male $(\mathrm{n},(\%))$ & $10023(62.8)$ & 1012 (82.5) & $9011(61.2)$ & $<0.001$ \\
\hline SBP (mm Hg) (SD) & 121.09 (15.85) & $131.78(16.47)$ & $120.19(15.47)$ & $<0.001$ \\
\hline TC (mmol/L) (SD) & $4.88(0.93)$ & $5.19(0.95)$ & $4.86(0.93)$ & $<0.001$ \\
\hline TG (mmol/L) (SD) & $1.46(1.10)$ & $2.18(1.49)$ & $1.40(1.04)$ & $<0.001$ \\
\hline $\mathrm{HDL}-\mathrm{C}(\mathrm{mmol} / \mathrm{L})(\mathrm{SD})$ & $1.26(0.25)$ & $1.15(0.22)$ & $1.27(0.25)$ & $<0.001$ \\
\hline LDL-C (mmol/L) (SD) & $2.92(0.78)$ & $3.20(0.80)$ & $2.90(0.77)$ & $<0.001$ \\
\hline FPG (mmol/L) (SD) & $5.06(1.04)$ & $5.51(1.61)$ & $5.03(0.97)$ & $<0.001$ \\
\hline
\end{tabular}

Continuous variables are presented as the means (SD).

*Obesity was defined as body mass index $(\mathrm{BMI}) \geq 28.0 \mathrm{~kg} / \mathrm{m}^{2}$

$\dagger P$ value for the difference of variables between the two data sets based on independent sample t-test or $\chi^{2}$ test.

$\ddagger$ The average number of health check-up for each participant is 2.62.

BUN, blood urea nitrogen; $\mathrm{Cr}$, creatinine; DBP, diastolic blood pressure; FPG, fasting plasma glucose; HDL-C, high-density lipoprotein cholesterol; LDL-C, low-density lipoprotein cholesterol; SBP, systolic blood pressure; SUA, serum uric acid; TC, total cholesterol; TG, triglycerides.

increased with the number of medical check-up years in the group with high SUA and normal SUA levels $(\mathrm{p}<0.001$ for trend) (figure 1). Finally, 1078 participants developed obesity over the 9-year period.

As presented in table 3, we observed at baseline significant differences on risk of obesity for SUA (per-1 OR=1.01 (95\% CI: 1.01 to 1.02$)$ ) or (per-SD OR=1.84 (95\% CI: 1.77 to 1.90$)$ ), age (OR=1.02 (95\% CI: 1.02 to 1.03$)$ ],

Table 2 The prevalence of obesity by gender, age of check-up stratified by baseline SUA

\begin{tabular}{cccc}
\hline \multirow{2}{*}{ Variable } & \multicolumn{2}{l}{ Obesity prevalence, $\mathbf{n}(\%)^{\star}$} & \\
\cline { 2 - 4 } Normal SUA & High SUA & P value \\
\hline Gender & & & \\
Male & $357 / 5280(6.8)$ & $570 / 3768(15.1)$ & $<0.001$ \\
Female & $104 / 4431(2.3)$ & $97 / 937(10.3)$ & $<0.001$ \\
Age group & & & \\
$<30$ & $88 / 3509(2.5)$ & $168 / 1643(10.2)$ & $<0.001$ \\
$30-44$ & $182 / 3736(4.9)$ & $309 / 1692(18.3)$ & $<0.001$ \\
$45-59$ & $121 / 1727(7.0)$ & $125 / 865(14.5)$ & $<0.001$ \\
$60-74$ & $54 / 606(8.9)$ & $53 / 378(14.0)$ & $<0.001$ \\
$\geq 75$ & $11 / 134(8.2)$ & $12 / 127(9.4)$ & $<0.001$ \\
Overall & $456 / 9711(4.6)$ & $669 / 4705(14.2)$ & $<0.001$ \\
\hline
\end{tabular}

High SUA level was defined as the SUA greater than $420 \mathrm{mmol} / \mathrm{L}$ in men and greater than $360 \mathrm{mmol} / \mathrm{L}$ in women, while the others are normal.

${ }^{*}$ Obesity prevalence $=$ (n of obesity) $/$ (total participants).

SUA, serum uric acid. and male ( $\mathrm{OR}=1.27$ (95\% CI: 1.16 to 1.39$)$ ) in the logistic regression analysis (Model 1). When converted to categorical analysis, the risks of obesity were greater among those with high level of SUA, men and younger participants. Likewise, with longitudinal data on the repeated medical checkups in the multivariable GEE model (Model 2 ), consistent risk factors for obesity were obtained. The estimates were observed as follows: (per-1 OR=1.01 (95\% CI: 1.01 to 1.02$)$ ) or (per-SD OR=1.85 (95\% CI: 1.77 to 1.91)) for SUA, OR=1.45 (95\% CI: 1.32 to 1.60) for men and OR=1.01 (95\% CI: 1.01 to 1.02) for age. In additional analysis by categorical variables, we observed similar results with higher risk in men and elderly participants.

As showed in table 4, similar results for GEE model analyses were observed in subgroup analyses. Significant associations between SUA and risk of obesity were observed, where women (per-SD OR=2.35 (2.16 to 2.55) ] and young participants (per-SD OR=1.87 (1.80 to 1.94)) had an elevated risk. We also performed analysis of baseline uric acid values versus obesity at the 9-year mark in men and women, respectively, where one eliminates baseline cases with hypertension, diabetes or elevated blood sugar, dyslipidaemia, normal kidney function, and baseline obesity. This result was consistent with the subgroup analysis and well validate the data.

To calculate the discrimination ability of SUA among obese participants at different times of medical check-up (1 to 8 ) or different years of medical check-up (2010 to 2018), ROC curves were calculated. Online supplemental figures 1 and 2 summarises the cut-off values and the area under receiver operating curves of SUA in obesity 
Table 3 Relationship between risk factors and risk of obesity in the models

\begin{tabular}{|c|c|c|c|c|}
\hline \multirow[b]{2}{*}{ Variable } & \multicolumn{2}{|l|}{ Model 1* } & \multicolumn{2}{|l|}{ Model 2† } \\
\hline & OR (95\% CI) & $P$ value & OR (95\% Cl) & P value \\
\hline \multicolumn{5}{|c|}{ Continuous analysis } \\
\hline Per-1 & 1.01 (1.01 to 1.02$)$ & $<0.001$ & 1.01 (1.01 to 1.02) & $<0.001$ \\
\hline Per-SD & 1.84 (1.77 to 1.90$)$ & $<0.001$ & 1.85 (1.77 to 1.91$)$ & $<0.001$ \\
\hline Female & Reference & & Reference & \\
\hline Male & 1.27 (1.16 to 1.39$)$ & $<0.001$ & $1.45(1.32$ to 1.60$)$ & $<0.001$ \\
\hline Age (years) & 1.02 (1.02 to 1.03$)$ & $<0.001$ & 1.01 (1.01 to 1.02$)$ & $<0.001$ \\
\hline \multicolumn{5}{|c|}{ Categorical analysis } \\
\hline \multicolumn{5}{|l|}{ SUA } \\
\hline \multicolumn{5}{|l|}{ Gender } \\
\hline Female & Reference & & Reference & \\
\hline Male & 1.25 (1.09 to 1.43$)$ & 0.002 & 1.69 (1.59 to 1.79$)$ & $<0.001$ \\
\hline \multicolumn{5}{|l|}{ Age group } \\
\hline$<30$ & Reference & & Reference & \\
\hline $30-44$ & 1.38 (1.14 to 1.66$)$ & 0.001 & $1.73(1.54$ to 1.91$)$ & $<0.001$ \\
\hline $45-59$ & 1.07 (0.89 to 1.30$)$ & 0.475 & 1.94 (1.72 to 2.18$)$ & $<0.001$ \\
\hline $60-74$ & $1.12(0.90$ to 1.38$)$ & 0.314 & 1.99 (1.72 to 2.32$)$ & $<0.001$ \\
\hline$\geq 75$ & $0.95(0.71$ to 1.27$)$ & 0.718 & 1.86 (1.50 to 2.31$)$ & $<0.001$ \\
\hline
\end{tabular}

*Model 1 was adjusted for the variables of SBP, DBP, TC, TG, HDL-C, LDL-C, FPG, BUN, Cr based on the first time of medical check-up participants by using multivariate logistic regression model (LRM).

†Model 2 was adjusted for the variables of repeated times or years of medical check-up, SBP, DBP, TC, TG, HDL-C, LDL-C, FPG, BUN, Cr based on all medical check-up participants by using generalised estimation equation model (GEE).

†High SUA level was defined as the SUA greater than $420 \mathrm{mmol} / \mathrm{L}$ in men and greater than $360 \mathrm{mmol} / \mathrm{L}$ in women, while the others are normal.

BUN, blood urea nitrogen; Cr, creatinine; DBP, diastolic blood pressure; FPG, fasting plasma glucose; HDL-C, high-density lipoprotein cholesterol; LDL-C, low-density lipoprotein cholesterol; SBP, systolic blood pressure; SUA, serum uric acid; TC, total cholesterol; TG, triglycerides.

participants stratified by gender. We found that the overall cut-off values of SUA were $429.5 \mu \mathrm{mol} / \mathrm{L}$ (range: $411.5-$ $488.5 \mu \mathrm{mol} / \mathrm{L}$ ) in men and $326.9 \mu \mathrm{mol} / \mathrm{L}$ (range: $298.5-$ $426.5 \mathrm{~mol} / \mathrm{L}$ ) in women when stratified by different times of medical checkups. Similarly, we calculated the overall cut-off values for SUA, which were $429.5 \mu \mathrm{mol} / \mathrm{L}$ (range: $366.7-431.5 \mu \mathrm{mol} / \mathrm{L}$ ) in men and $326.9 \mu \mathrm{mol} / \mathrm{L}$ (range: $301.5-362.1 \mu \mathrm{mol} / \mathrm{L}$ ) in women when stratified by different years of medical checkups.

\section{DISCUSSION}

To the best of our knowledge, this is the first longitudinal study that estimated the relationship between SUA and obesity over a long time period in China. The prevalence of obesity was approximately $14.2 \%$ for high SUA level. Previous studies found that the prevalence of hyperuricaemia ranged from $2.5 \%$ to $25 \%$ depending on the study population country. ${ }^{18}$ For instance, the prevalence rates were reported to be $5 \%$ in the Caucasus and $24.4 \%$ in Thailand. ${ }^{19}{ }^{20}$ Overall, we found high SUA level was associated with increased risk of obesity, within $O R$ value of 1.85 (95\% CI: 1.77 to 1.91 ) in the GEE model for all participants, which was nearly consistent with prior studies. ${ }^{21}{ }^{22}$ Currently, obesity and hyperuricaemia, as well as their associated health complications (eg, metabolic syndrome) have emerged as a major public health concern as a result of the growing prevalence, and the estimated economic burden.?

Several recent studies have investigated the mechanism of SUA on increasing the risk of obesity, suggesting the influence of overproduction and poor renal excretion. ${ }^{23}$ Prior studies reported that increased SUA level is closely related to excessive production of UA, and the reduction of urinary uric acid excretion and clearance. ${ }^{24}$ This ultimately leads to increased risk of patients with visceral fatty obesity. ${ }^{23}$ Visceral fat accumulation (VFA) results in 
Table 4 Relationship between risk factors and risk of obesity in the models stratified by gender and age group

\begin{tabular}{|c|c|c|c|c|}
\hline \multirow{3}{*}{$\begin{array}{l}\text { Variable } \\
\text { Gender }\end{array}$} & \multicolumn{4}{|c|}{ Generalised estimation equation model (GEE)* } \\
\hline & \multicolumn{2}{|l|}{ Male } & \multicolumn{2}{|l|}{ Female } \\
\hline & OR (95\% Cl) & $P$ value & OR (95\% Cl) & $P$ value \\
\hline \multicolumn{5}{|l|}{ SUA ( $\mu \mathrm{mol} / \mathrm{L})$} \\
\hline Per-1 & 1.01 (1.01 to 1.02$)$ & $<0.001$ & 1.01 (1.01 to 1.02$)$ & $<0.001$ \\
\hline \multicolumn{5}{|c|}{ Categorical variables } \\
\hline \multicolumn{5}{|l|}{ SUA } \\
\hline Normal SUA & Reference & & Reference & \\
\hline High SUA & 2.40 (2.23 to 2.59$)$ & $<0.001$ & 3.79 (3.23 to 4.45$)$ & $<0.001$ \\
\hline \multicolumn{5}{|l|}{ SUA ( $\mu \mathrm{mol} / \mathrm{L})$} \\
\hline Per-1 & 1.01 (1.01 to 1.02$)$ & $<0.001$ & $1.00(1.00$ to 1.01$)$ & $<0.001$ \\
\hline Per-SD & $1.87(1.80$ to 1.94$)$ & $<0.001$ & $1.48(1.34$ to 1.62$)$ & $<0.001$ \\
\hline \multicolumn{5}{|c|}{ Categorical variables } \\
\hline \multicolumn{5}{|l|}{ SUA } \\
\hline Normal SUA & Reference & & Reference & \\
\hline High SUA & 2.78 (2.58 to 2.99 ) & $<0.001$ & 1.99 (1.63 to 2.43 ) & $<0.001$ \\
\hline
\end{tabular}

*Model was adjusted for the variables of repeated times or years of medical check-up, age, sex, SBP, DBP, TC, TG, HDL-C, LDL-C, FPG, BUN, Cr based on all medical check-up participants by using GEE.

†High SUA level was defined as the SUA greater than $420 \mathrm{mmol} / \mathrm{L}$ in men and greater than $360 \mathrm{mmol} / \mathrm{L}$ in women, while the others are normal.

BUN, blood urea nitrogen; Cr, creatinine; DBP, diastolic blood pressure; FPG, fasting plasma glucose; HDL-C, high-density lipoprotein cholesterol; LDL-C, low-density lipoprotein cholesterol; SBP, systolic blood pressure; SUA, serum uric acid; TC, total cholesterol; TG, triglycerides.

a large influx of plasma free fatty acids into the portal vein and liver. This stimulates the synthesis of TG and subsequently produced large amounts of uric acid (UA) through the activated UA synthesis pathway. ${ }^{25}{ }^{26}$ Additionally, many researchers have reported a significant correlation between VFA and BMI. ${ }^{2728}$ Therefore, because of the close biological relationship between UA and BMI, it is of great importance for preventive medicine to closely examine the interaction between UA and BMI.

Conflicting results regarding gender and age differences for the association between SUA and obesity have been reported. ${ }^{10}{ }^{29}$ Our study observed significant differences in obesity participants with elevated OR value among high SUA level, men and elderly for all medical check-up participants. A similar study reported a positive relationship between BMI and SUA levels among healthy individuals in China. ${ }^{30}$ Nevertheless, in this study the subgroup analyses showed that significant associated risk between SUA and obesity were observed higher in women and young participants. This is consistent with a Thailand study that reported high SUA concentrations were associated with greater risk of obesity in women. ${ }^{31}$ However, studies in Bangladesh and Japan reported that elevated SUA predicted obesity higher in men and the elderly. ${ }^{8291}$ Perhaps the associations of SUA with obesity varies by populations. Moreover, in a 10-year follow-up study, BMI was observed to significantly increase with higher SUA levels regardless of race and gender. ${ }^{32}$ Therefore, greater attention should be provided to those vulnerable populations in clinical guidelines.

An important observation was that the association between SUA and risk of obesity in the LRM (OR=1.84 (95\% CI: 1.77 to 1.90)) for data at baseline was nearly consistent with the analyses in the GEE model (OR=1.85 (95\% CI: 1.77 to 1.91)) for 9-year all participants. The risk of obesity within hyperuricaemia remained stable over the years. Therefore, shortterm medical check-up results can reflect the development of chronic diseases. ${ }^{33}$ Regarding the assessment of cut-off values from ROC of SUA in obesity participants, the cut-off values of SUA were $429.5 \mu \mathrm{mol} / \mathrm{L}$ in men and $326.9 \mu \mathrm{mol} / \mathrm{L}$ in women in stratified analysis by times or years of medical check-up. The cut-off value was approximately consistent with the international standard for men. ${ }^{34}$ However, it was underestimated for women in the group of obese participants. Perhaps the proportion of women were fewer in this study. The cut-off values for SUA in the study may be useful for distinguishing tests among obese and non-obese participants, which were significant for certain risk value prediction and guidance. ${ }^{35}$ 
To our knowledge, we must note several limitations in the present study. First, the underlying mechanism by which SUA is increased in obese individuals remains not well understood. Second, this study did not collect information on whether participants were prescribed medication to treat hyperuricaemia. Additionally, some medications used to treat hypertension may increase uric acid levels. Third, there are numerous confounding factors that have not been considered, which can be studied together with questionnaires in the future. Moreover, the younger screening population in this study may underestimate the increased risk of uric acid among the elderly obese.

The present study has several strengths that must be noted. First, to our knowledge this is the first large long-term medical check-up study to explore the relationship between SUA and obesity in China. Second, the study analysis was based on the GEE model with high quality data by controlling for confounding factors, which can increase the accuracy of the prediction. Third, participants were representative of the general population with regard to clinical check-up and obesity status, enhancing the generalisability of our findings. Moreover, results from this study could inform prevention methods for obesity, especially in medically underserved areas where medical service is insufficient.

This study filled current gaps in literature by analysing the relationship between SUA and obesity using medical check-up data. We observed that medical check-up data can be used to improve the risk of obesity prediction accuracy. The medical check-up data used in this study can help provide information that will facilitate intervention development and adoption at the individual level. ${ }^{36}$ The utility of medical check-up data can potentially reach beyond predictive power alone in the near future.

\section{CONCLUSIONS}

In conclusion, our study observed significant associations between SUA and obesity in this 9-year longitudinal study. We mainly found higher SUA level was associated with increased risk of obesity. The prevalence of obesity was approximately $14.2 \%$ and significantly increased with the number of medical check-up years in the group with high level of SUA. Additionally, the increased risk of obesity was greater for high SUA level, men and elderly participants. Subgroup analyses revealed significant associations between SUA and obesity with higher risk for women and young participants. Additionally, the cut-off for SUA on risk of obesity were approximately consistent with the international standard. More evidence from well-designed studies are needed to confirm our findings.

\section{Author affiliations}

${ }^{1}$ Center for Clinical Epidemiology and Methodology, Guangdong Second Provincial General Hospital, Guangdong, China

${ }^{2}$ Institute of Ultrasound in Musculoskeletal Sports Medicine, Guangdong Second Provincial General Hospital, Guangzhou, China

${ }^{3}$ Department of Epidemiology and Biostatistics, University at Albany State University of New York, Albany, New York, USA
${ }^{4}$ Institute for Environmental and Climate Research, Jinan University, Guangzhou, China

${ }^{5}$ Guangdong Traditional Medical and Sports Injury Rehabilitation Research Institute, Guangdong Second Provincial General Hospital, Guangzhou, China

${ }^{6}$ Center for Information, Guangdong Second Provincial General Hospital, Guangzhou, China

${ }^{7}$ Center for Health Management and Examination, Guangdong Second Provincial General Hospital, Guangzhou, China

${ }^{8}$ Department of Ultrasound, Guangdong Second Provincial General Hospital, Guangzhou, China

${ }^{9}$ Department of Health Research Methods, Evidence, and Impact (HEI), McMaster University, Hamilton, Ontario, Canada

Contributors Guowei Li, Jie Zeng, Guanming Li: conceived and designed the study. Guowei Li, Jie Zeng, Wayne R Lawrence, Jun Yang: acquired data, performed statistical analyses and interpretation, and drafted the manuscript. Junzhang Tian, Cheng Li, Wanmin Lian, Jingjun He, Hongying Qu, Xiaojie Wang, Hongmei Liu: provided professional and statistical support, and made several critical revisions to the manuscript. All authors read and approved the final manuscript.

Funding Research grants from the Science Foundation of Guangdong Second Provincial General Hospital (YQ2019-008; Dr Jie Zeng), the Science and Technology Programme of Guangzhou (202002030252; Dr Guowei Li) and the Science Foundation of Guangdong Second Provincial General Hospital (YY2018-002; Dr Guowei Li).

Map disclaimer The depiction of boundaries on this map does not imply the expression of any opinion whatsoever on the part of BMJ (or any member of its group) concerning the legal status of any country, territory, jurisdiction or area or of its authorities. This map is provided without any warranty of any kind, either express or implied.

Competing interests None declared.

Patient consent for publication Not required.

Ethics approval The study was approved by Guangdong Second Provincial General Hospital Ethics Committee (reg. no. 20190717-01(2)-YXKXYJ-KT). The need for consent was waived due to the retrospective nature of this study.

Provenance and peer review Not commissioned; externally peer reviewed.

Data availability statement All data relevant to the study are included in the article or uploaded as supplementary information.

Supplemental material This content has been supplied by the author(s). It has not been vetted by BMJ Publishing Group Limited (BMJ) and may not have been peer-reviewed. Any opinions or recommendations discussed are solely those of the author(s) and are not endorsed by BMJ. BMJ disclaims all liability and responsibility arising from any reliance placed on the content. Where the content includes any translated material, BMJ does not warrant the accuracy and reliability of the translations (including but not limited to local regulations, clinical guidelines, terminology, drug names and drug dosages), and is not responsible for any error and/or omissions arising from translation and adaptation or otherwise.

Open access This is an open access article distributed in accordance with the Creative Commons Attribution Non Commercial (CC BY-NC 4.0) license, which permits others to distribute, remix, adapt, build upon this work non-commercially, and license their derivative works on different terms, provided the original work is properly cited, appropriate credit is given, any changes made indicated, and the use is non-commercial. See: http://creativecommons.org/licenses/by-nc/4.0/.

ORCID iD

Jie Zeng http://orcid.org/0000-0002-3389-320X

\section{REFERENCES}

1 Nagahama S, Kashino I, Hu H, et al. Haemoglobin A1c and hearing impairment: longitudinal analysis using a large occupational health check-up data of Japan. BMJ Open 2018;8:e023220.

$2 \mathrm{Kim}$ YJ, Park H. Improving prediction of high-cost health care users with medical Check-Up data. Big Data 2019;7:163-75.

3 Vos T, Barber RM, Bell B, et al. Global, regional, and national incidence, prevalence, and years lived with disability for 301 acute and chronic diseases and injuries in 188 countries, 1990-2013: a 
systematic analysis for the global burden of disease study 2013. The Lancet 2015;386:743-800.

$4 \mathrm{Ng} \mathrm{M}$, Fleming T, Robinson M, et al. Global, regional, and national prevalence of overweight and obesity in children and adults during 1980-2013: a systematic analysis for the global burden of disease study 2013. The Lancet 2014;384:766-81.

5 Liu R, Han C, Wu D, et al. Prevalence of hyperuricemia and gout in mainland China from 2000 to 2014: a systematic review and metaanalysis. Biomed Res Int 2015;2015:1-12.

6 Ishizaka N, Ishizaka Y, Toda A, et al. Changes in waist circumference and body mass index in relation to changes in serum uric acid in Japanese individuals. J Rheumatol 2010;37:410-6.

7 Chen M-Y, Zhao C-C, Li T-T, et al. Serum uric acid levels are associated with obesity but not cardio-cerebrovascular events in Chinese inpatients with type 2 diabetes. Sci Rep 2017;7:40009.

8 Dai X, Yuan J, Yao P, et al. Association between serum uric acid and the metabolic syndrome among a middle- and old-age Chinese population. Eur J Epidemiol 2013;28:669-76.

9 Johnson RJ, Lanaspa MA, Gaucher EA. Uric acid: a danger signal from the RNA world that may have a role in the epidemic of obesity, metabolic syndrome, and cardiorenal disease: evolutionary considerations. Semin Nephrol 2011;31:394-9.

10 Ali N, Perveen R, Rahman S, et al. Prevalence of hyperuricemia and the relationship between serum uric acid and obesity: a study on Bangladeshi adults. PLoS One 2018;13:e0206850.

11 Yang C, Yang S, Feng C, et al. Associations of hyperuricemia and obesity with remission of nonalcoholic fatty liver disease among Chinese men: a retrospective cohort study. PLoS One 2018;13:e0192396.

12 Sui X, Church TS, Meriwether RA, et al. Uric acid and the development of metabolic syndrome in women and men. Metabolism 2008;57:845-52.

13 You L, Liu A, Wuyun G, et al. Prevalence of hyperuricemia and the relationship between serum uric acid and metabolic syndrome in the Asian Mongolian area. J Atheroscler Thromb 2014;21:355-65.

14 WHO Expert Consultation. Appropriate body-mass index for Asian populations and its implications for policy and intervention strategies. Lancet 2004;363:157-63.

15 Li M-F, Ren Y, Zhao C-C, et al. Prevalence and clinical characteristics of lower limb atherosclerotic lesions in newly diagnosed patients with ketosis-onset diabetes: a cross-sectional study. Diabetol Metab Syndr 2014;6:71.

16 Bůzková P, Brown ER, John-Stewart GC. Longitudinal data analysis for generalized linear models under participant-driven informative follow-up: an application in maternal health epidemiology. Am J Epidemiol 2010;171:189-97.

17 Habibzadeh F, Habibzadeh P, Yadollahie M. On determining the most appropriate test cut-off value: the case of tests with continuous results. Biochem Med 2016;26:297-307.

18 Remedios C, Shah M, Bhasker AG, et al. Hyperuricemia: a reality in the Indian obese. Obes Surg 2012;22:945-8.

19 Uaratanawong S, Suraamornkul S, Angkeaw S, et al. Prevalence of hyperuricemia in Bangkok population. Clin Rheumatol 2011;30:887-93.
20 Ford DK, Demos AM. Serum uric acid levels of healthy Caucasian, Chinese and Haida Indian males in British Columbia. Can Med Assoc J 1964;90:1295-7.

21 Kuwabara M, Kuwabara R, Hisatome I, et al. "Metabolically Healthy" Obesity and Hyperuricemia Increase Risk for Hypertension and Diabetes: 5-year Japanese Cohort Study. Obesity 2017:25:1997-2008.

22 Zhang N, Chang Y, Guo X, et al. A body shape index and body Roundness index: two new body indices for detecting association between obesity and hyperuricemia in rural area of China. Eur $J$ Intern Med 2016;29:32-6.

23 Matsuura F, Yamashita S, Nakamura T, et al. Effect of visceral fat accumulation on uric acid metabolism in male obese subjects: visceral fat obesity is linked more closely to overproduction of uric acid than subcutaneous fat obesity. Metabolism 1998;47:929-33.

24 Han T, Meng X, Shan R, et al. Temporal relationship between hyperuricemia and obesity, and its association with future risk of type 2 diabetes. Int J Obes 2018;42:1336-44.

25 Fabregat I, Revilla E, Machado A. Short-Term control of the pentose phosphate cycle by insulin could be modulated by the NADPH/ NADP ratio in rat adipocytes and hepatocytes. Biochem Biophys Res Commun 1987;146:920-5.

26 Fox IH. Metabolic basis for disorders of purine nucleotide degradation. Metabolism 1981;30:616-34.

27 Examination Committee of Criteria for 'Obesity Disease' in Japan, Japan Society for the Study of Obesity. New criteria for 'obesity disease' in Japan. Circ J 2002;66:987-92.

28 Oka R, Miura K, Sakurai M, et al. Comparison of waist circumference with body mass index for predicting abdominal adipose tissue. Diabetes Res Clin Pract 2009;83:100-5

29 Tanaka K, Ogata S, Tanaka H, et al. The relationship between body mass index and uric acid: a study on Japanese adult twins. Environ Health Prev Med 2015;20:347-53.

30 Wang $\mathrm{H}$, Wang $\mathrm{L}$, Xie $\mathrm{R}$, et al. Association of serum uric acid with body mass index: a cross-sectional study from Jiangsu Province, China. Iran J Public Health 2014;43:1503-9.

31 Jaipakdee J, Jiamjarasrangsri W, Lohsoonthorn V, et al. Prevalence of metabolic syndrome and its association with serum uric acid levels in Bangkok Thailand. Southeast Asian J Trop Med Public Health 2013;44:512-22.

32 Rathmann W, Haastert B, lcks A, et al. Ten-Year change in serum uric acid and its relation to changes in other metabolic risk factors in young black and white adults: the cardia study. Eur J Epidemiol 2007;22:439-45.

33 Nohara Y, Kai E, Ghosh PP, et al. Health checkup and telemedical intervention program for preventive medicine in developing countries: verification study. J Med Internet Res 2015;17:e2.

34 Bardin T, Richette P. Definition of hyperuricemia and gouty conditions. Curr Opin Rheumatol 2014;26:186-91.

35 Mongioì LM, Condorelli RA, Barbagallo $\mathrm{F}$, et al. Accuracy of the low-dose ACTH stimulation test for adrenal insufficiency diagnosis: a re-assessment of the cut-off value. J Clin Med 2019;8:806.

36 Taninaga J, Nishiyama Y, Fujibayashi K, et al. Prediction of future gastric cancer risk using a machine learning algorithm and comprehensive medical check-up data: a case-control study. Sci Rep 2019;9:12384. 
Association between serum uric acid and obesity in Chinese adults: a 9-year longitudinal data analysis

Zeng J, Lawrence WR,Yang J, et al. Association between serum uric acid and obesity in Chinese adults: a 9-year longitudinal data analysis. BMJ Open 2021;11:e041919. doi: 10.1136/bmjopen-2020-041919.

This article was previously published with an error. The funding information in the published article was incomplete. The updated funding information is stated below:

Research grants from the Science Foundation of Guangdong Second Provincial General Hospital (YQ2019-008; Dr Jie Zeng), the Science and Technology Programme of Guangzhou (202002030252; Dr Guowei Li) and the Science Foundation of Guangdong Second Provincial General Hospital (YY2018-002; Dr Guowei Li).

Open access This is an open access article distributed in accordance with the Creative Commons Attribution Non Commercial (CC BY-NC 4.0) license, which permits others to distribute, remix, adapt, build upon this work non-commercially, and license their derivative works on different terms, provided the original work is properly cited, appropriate credit is given, any changes made indicated, and the use is non-commercial. See: http://creativecommons.org/licenses/by-nc/4.0/.

(c) Author(s) (or their employer(s)) 2021. Re-use permitted under CC BY-NC. No commercial re-use. See rights and permissions. Published by BMJ.

BMJ Open 2021;11:e041919corr1. doi:10.1136/bmjopen-2020-041919corr1

Check for updates 